Supporting information

\title{
Polymeric Self-assembly into Micelles and Hollow Spheres with Multi-scale Cavities Driven by Inclusion Complexation
}

\author{
Jing Wang, Ming Jiang*
}

\section{Synthesis}

Scheme S1. The synthesis procedures of the monomers and polymers.
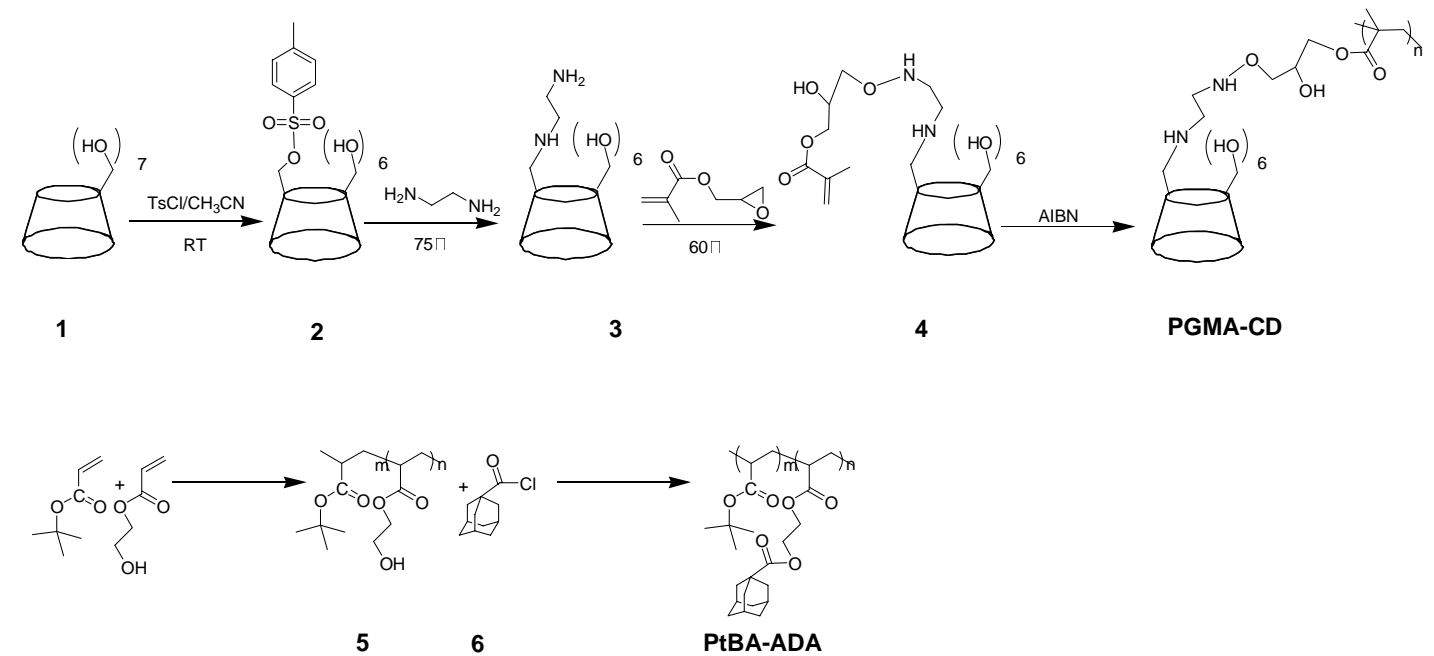

The difficulties in preparing such linear polymers carrying $\beta$-CD side groups are associated with the synthesis of monomers with mono-vinyl substitution of $\beta-\mathrm{CD}$. A CD carrying multiple vinyl groups, which is easily produced, leads to highly branched or crosslinked polymers. In this work by carefully controlling the reaction conditions from both $\mathbf{1}$ to $\mathbf{2}$ and $\mathbf{3}$ to $\mathbf{4}$, we succeeded in obtaining mono-substituted monomer 4 (Scheme 1), which was confirmed by MALDI-TOF measurements.

\section{TsCD (Mono-6-(p-tolylsulfonyl)- $\beta$-cyclodextrin) (2)}

TsCD was synthesized as that reported in ref[1]. MALDI-TOF on THAP matrix (M-OTs + $\left.\mathrm{Na}^{+}\right) \boldsymbol{m} / z$ calcd for $\mathrm{C}_{49} \mathrm{H}_{76} \mathrm{NaO}_{37} \mathrm{~S}\left(\mathrm{M}-\mathrm{OTs}+\mathrm{Na}^{+}\right.$) 1311.37, measured 1311.34 (Figure S1a). ${ }^{1} \mathrm{H}$ NMR supported the mono-modified structure as well.

EDA-CD (3)

EDA-CD was synthesized as that reported in ref[2], MALDI-TOF on THAP matrix $\left(\mathrm{M}+\mathrm{Na}^{+}\right)$ $\boldsymbol{m} / \boldsymbol{z}$ calcd for $\mathrm{C}_{44} \mathrm{H}_{76} \mathrm{~N}_{2} \mathrm{NaO}_{34}\left(\mathrm{M}-\mathrm{OTs}+\mathrm{Na}^{+}\right)$1199.42, measured 1199.99(Figure S1b).

GMA-CD (4)

GMA-CD was synthesized by the reaction of $\mathbf{3}$ and glycidyl methacrylate (GMA)[2]. According to the recipe in [2], i.e. using excess GMA we got mulit-substituted CD monomer. Instead, when just 1 equiv GMA was used, mono-substituted GMA-CD was obtained as proved by MALDI-TOF on THAP matrix. $\boldsymbol{m} / z$ calcd for $\mathrm{C}_{49} \mathrm{H}_{82} \mathrm{~N}_{2} \mathrm{NaO}_{38}\left(\mathrm{M}+\mathrm{Na}^{+}\right)$1329.44, measured 1329.7 (Figure S1c). 


\section{Polymerization of GMA-CD}

$0.15 \mathrm{~g}$ GMA-CD and $20 \mathrm{mg}$ AIBN were first dissolved in DMF, and then after purged with nitrogen for $30 \mathrm{~min}$ the solution was heated to $75{ }^{\circ} \mathrm{C}$. The solution turned highly viscous after 20 $\min$. The reaction proceeded for $3 \mathrm{~h}$ and then was stopped by diluting with DMF followed by precipitation in acetone. The molecular weight $\left(1.04 \times 10^{4}\right)$ was determined by Size Exclusion Chromatography (SEC) using PS standard and DMF as eluent.

\section{Preparation of PtBA -HEA and PtBA -ADA}

$\mathrm{CuBr}\left(29.5 \mathrm{mg}, 2.0625 \times 10^{-4} \mathrm{~mol}\right)$ was added to a schlenk tube which was then sealed with a rubber stopper. The schlenk tube was degassed and filled with nitrogen three times, and kept filled up with nitrogen. tert-butyl acrylate (t-BA) $\left(3 \mathrm{~mL}, 2.025 * 10^{-2} \mathrm{~mol}\right)$, 2-hydroxyethyl acrylate (HEA) $\left(0.21 \mathrm{~mL}, 2.025 \times 10^{-3} \mathrm{~mol}\right)$, anisole $0.75 \mathrm{~mL}$ and $\mathrm{N}, \mathrm{N}, \mathrm{N}^{\prime}, \mathrm{N}^{\prime \prime}, \mathrm{N}^{\prime}-$ Pentamethyldiethylenetriamine (PMDETA) $(42 \mu \mathrm{L})$ were added into the tube followed by a three-time-frozen-thaw cycle. After adding methyl 2-bromopropionate (MBP) $\left(22 \mu \mathrm{L}, 2.0625 \times 10^{-5} \mathrm{~mol}\right)$ the tube was placed in an oil bath thermostated at $65{ }^{\circ} \mathrm{C}$. $8 \mathrm{~h}$ later, the solution was quenched in liquid nitrogen and diluted by excess THF. To remove the copper catalyst the solution was filtered through a column of alumina. The solution was concentrated and then precipitated to excess $\mathrm{MeOH} / \mathrm{H}_{2} \mathrm{O}(1 / 1, \mathrm{v} / \mathrm{v})$ solution. After twice precipitation-dissolving the polymer power was dried in vacuum. The polymer had Mn $1.67 \times 10^{4}$ and $\mathrm{Mw} / \mathrm{Mn}=1.21$ measured by SEC using PS standard and THF as eluent. The product was characterized by ${ }^{1} \mathrm{H}$ NMR (Figure S2a).

The conversion of the PtBA-HEA of the copolymer to PtBA-ADA was accomplished by mixing polymer 5 with 2 molar equiv of 1-adamantanecarbonyl chloride $\mathbf{6}$ in dry pyridine at room temperature overnight. The polymer solution was filtered and washed by THF, and then the filtrate was concentrated and added to excess $\mathrm{MeOH} / \mathrm{H}_{2} \mathrm{O}(1 / 1, \mathrm{v} / \mathrm{v})$ to precipitate out PtBA - ADA. The polymer power was dried under vacuum. The polymer had $\mathrm{Mn} 1.87 \times 10^{4}$ and $\mathrm{Mw} / \mathrm{Mn}=1.28$ measured by SEC using PS standard and THF as eluent. The product was characterized by ${ }^{1} \mathrm{H}$ NMR (Figure S2b).

\section{Micellization}

To prepare the micellar solutions, PtBA-ADA and PGMA-CD was firstly dissolved in DMF and mixed together, and then calculated volume of water was added dropwise into the mixture. Typically for M3 preparation, $0.05 \mathrm{~mL}$ PtBA-ADA in DMF $\left(0.01 \mathrm{~g} \bullet \mathrm{mL}^{-1}\right)$ was mixed with $0.05 \mathrm{~mL}$ PtBA-ADA $\left(0.01 \mathrm{~g} \bullet \mathrm{mL}^{-1}\right)$ and another $0.05 \mathrm{~mL} \mathrm{DMF}$ under ultrasonification, and then $6 \mathrm{~mL}$ water was added dropwise over $10 \mathrm{~min}$. The solution turned bluish which indicated the formation of aggregates. All the solutions had the same PtBA-ADA concentration of $1.25 \times 10^{-5} \mathrm{~g} \cdot \mathrm{mL}^{-1}$, the concentration of PGMA-CD depended on the desired weight ratio of PtBA-ADA to PGMA-CD. In the final solution the volume ratio of water to DMF is $40 / 1$.

For zeta-potential measurements, $20 \mathrm{mg}$ ADA-ca (in the form of potassium salt) or ADA-amine was added into $10 \mathrm{~mL}$ micellar solution and stirred overnight.

In FLS experiments, $3 \mathrm{~mL}$ of the micellar solution with added ANS was stirred for $24 \mathrm{~h}$ and under ultrasonic condition for $20 \mathrm{~min}$ before measurements. The final ANS concentration is 0.05 $\mathrm{mM}$.

\section{Hollow sphere preparation}

For M3 shell crosslinking, $3 \mathrm{~mL}$ micellar solution was mixed with $10 \mu \mathrm{L}$ glutaraldehyde (GA) under stirring and kept overnight. Then the solution was concentrated under reduced pressure at 
room temperature and then transferred to $3 \mathrm{~mL}$ DMF. One-day stirring at $50{ }^{\circ} \mathrm{C}$ was performed for swelling the core, de-complexation between $\beta-\mathrm{CD}$ and $\mathrm{ADA}$ and removal of the core.

\section{Measurements}

A commercial laser light scattering (LLS) spectrometer (Malvern Autosizer 4700) equipped with a multi- $\tau$ digital time correlation (Malvern PCS7132) and a solid-state laser (ILT 5500QSL, $100 \mathrm{~mW}$ output at $\lambda_{0}=532 \mathrm{~nm}$ ) and a CONTIN program are used. All the DLS measurements were done at $25.0 \pm 0.1{ }^{\circ} \mathrm{C}$. TEM observations were performed on a Philips CM 120 microscope at an accelerating voltage of $80 \mathrm{KV}$. The specimens were prepared by depositing $10 \mu \mathrm{L}$ of aggregates solution on carbon-coated grids. AFM imaging was performed in tapping mode on a Nanoscope IV from Digital Instruments, the sample preparation was similar with that for TEM but mica was used as substrate. The $\zeta$-potentials of the aggregates were measured on Zetasize nano ZS90 (Malvern, UK). The fluorescence was observed by using a fluorescence spectrophotometer FLS-920 (Edinberg Instruments). The excitation wavelength was $370 \mathrm{~nm}$, while the emission wavelength was recorded from 400 to $700 \mathrm{~nm}$. The spectral resolution of emission was $1 \mathrm{~nm}$. ${ }^{1} \mathrm{H}$ NMR $(400 \mathrm{MHz})$ spectra were performed on a Varian Mercury Plus 400 with tetramethylsilane as the internal standard. NOE spectra were measured using pulse sequences and standard procedures offered by Varian. The NOE difference spectrum was attained by subtracting the FID (free induction decay) data from that of control experiment obtained by irradiation at a no-resonance area. MALDI experiments were carried out using a Shimadzu AXIMA-CFR ${ }^{\mathrm{TM}}$ plus time-of-flight mass spectrometer (Kratos Analytical, Manchester, U.K.). A typical procedure to prepare the sample: $2 \mu \mathrm{L}$ of sample solution in DMF $\left(10 \mathrm{mg}^{\circ} \mathrm{mL}^{-1}\right), 2 \mu \mathrm{L}$ of NaI solution in DMF $\left(10 \mathrm{mg} \cdot \mathrm{mL}^{-1}\right)$ and $20 \mu \mathrm{L}$ of matrix solution in DMF (2',4',6'-Trihydroxyacetophenone Monohydrate, THAP, 10 $\mathrm{mg} \cdot \mathrm{mL}^{-1}$ ) were mixed. Then $2 \mu \mathrm{L}$ of the sample mixture was applied to the sample target and air-dried. The spectra were acquired as the follow conditions: a nitrogen laser emitting at $337 \mathrm{~nm}$, $120 \mu \mathrm{J}$ laser power and at least 512 scans. Reduced solution viscosity measurements were made with an Ubbelodhe viscometer at 25,35 and $50^{\circ} \mathrm{C}$ in DMF with a total polymer concentration of 2.0 $\mathrm{mg} \cdot \mathrm{mL}^{-1}$. The samples with different compositions were prepared by mixing solutions of PGMA-CD and PtBA-ADA as desired. Specific viscosity measurements were made at $30^{\circ} \mathrm{C}$. Molecular weights are measured by SEC at Agilent1100 using PS standard with DMF as eluent for PGMA-CD and THF for PtBA-ADA polymers. 


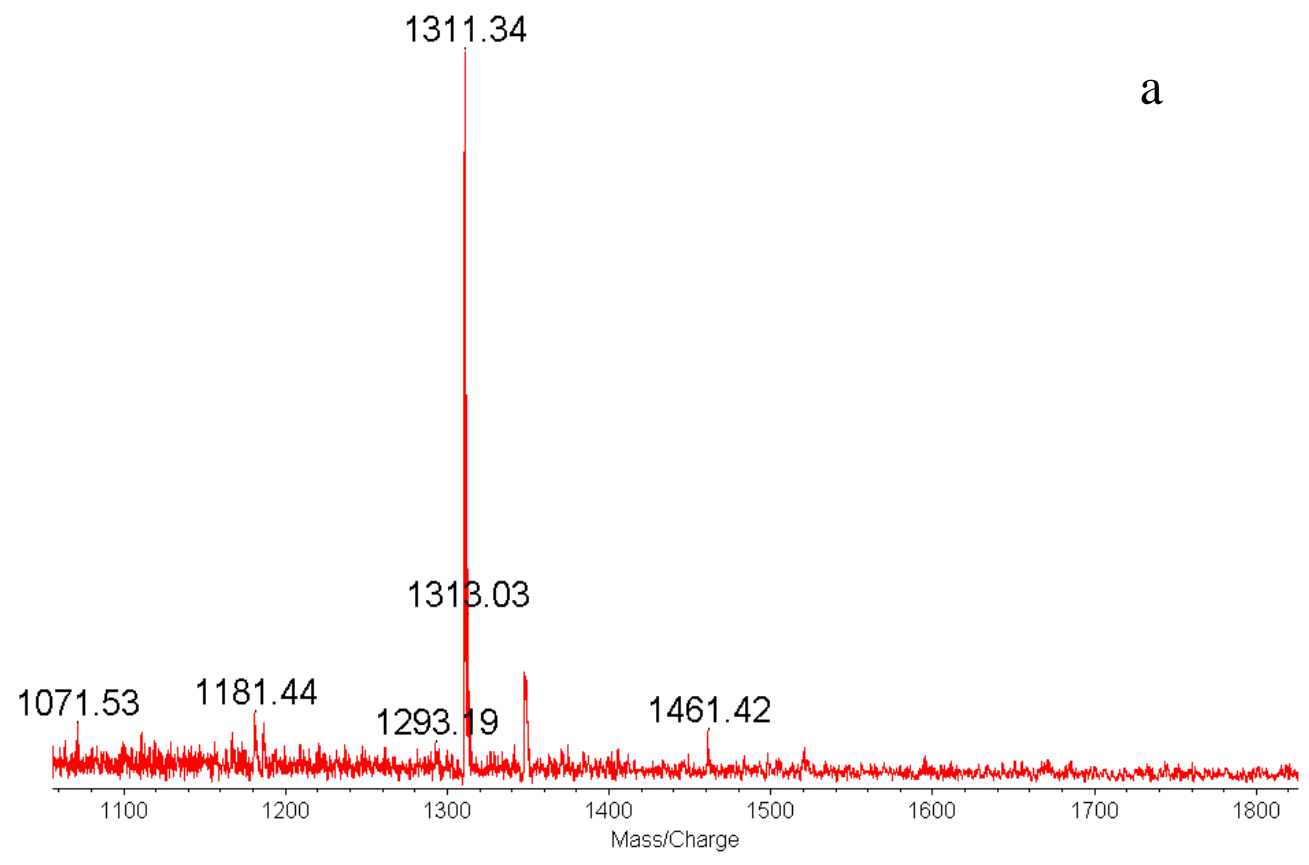

1199.99

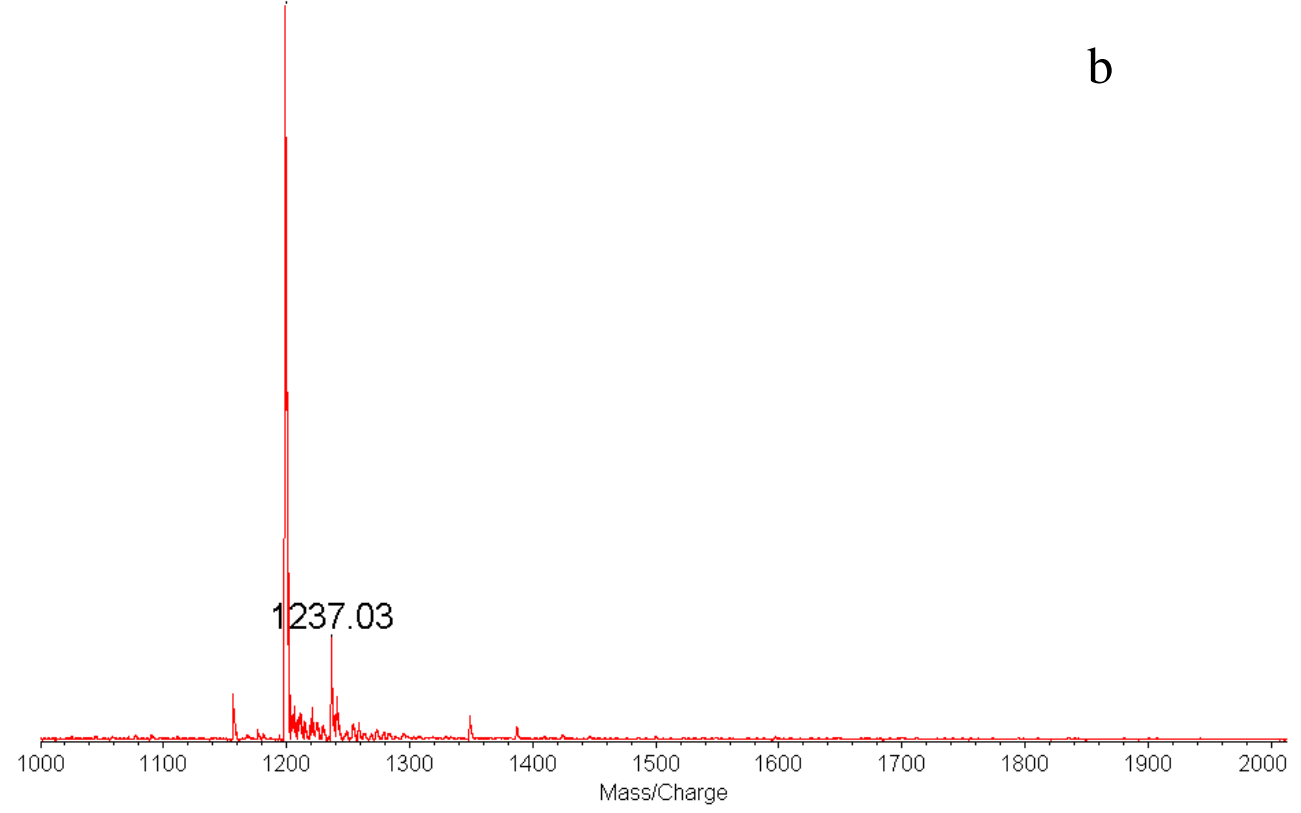




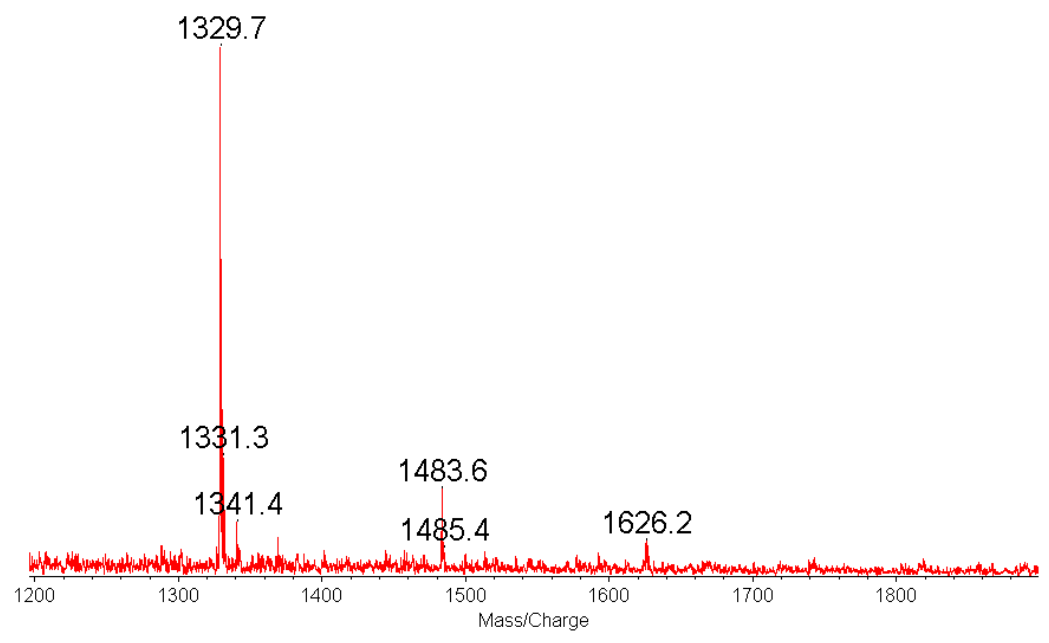

C

Figure S1. a,b,c) MALDI TOF spectra of TsCD (a), EDA-CD (b) and GMA-CD (c)

a

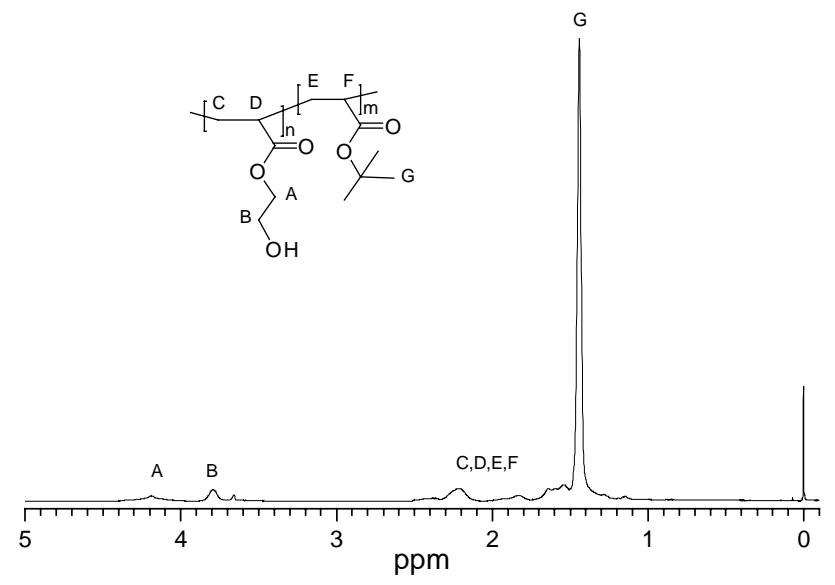


b

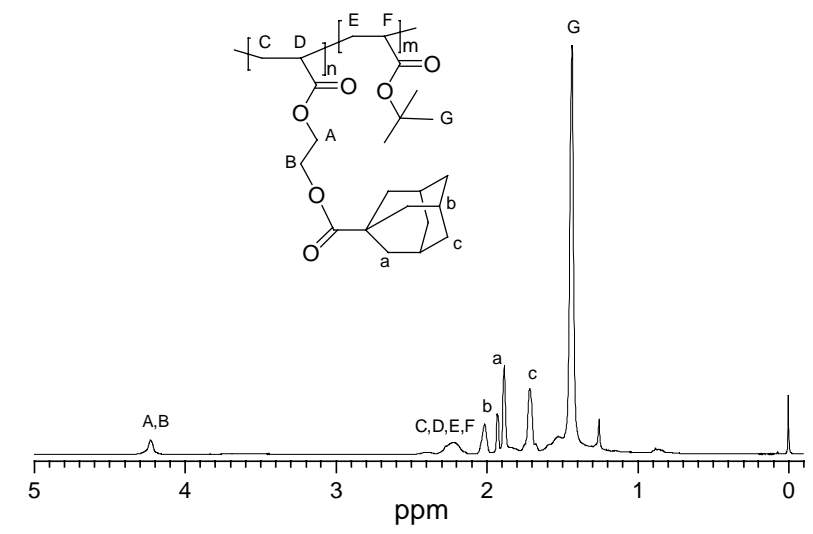

Figure S2 a,b) ${ }^{1} \mathrm{H}$ NMR spectra of PtBA-HEA (a) and PtBA-ADA (b) in $\mathrm{CDCl}_{3}$

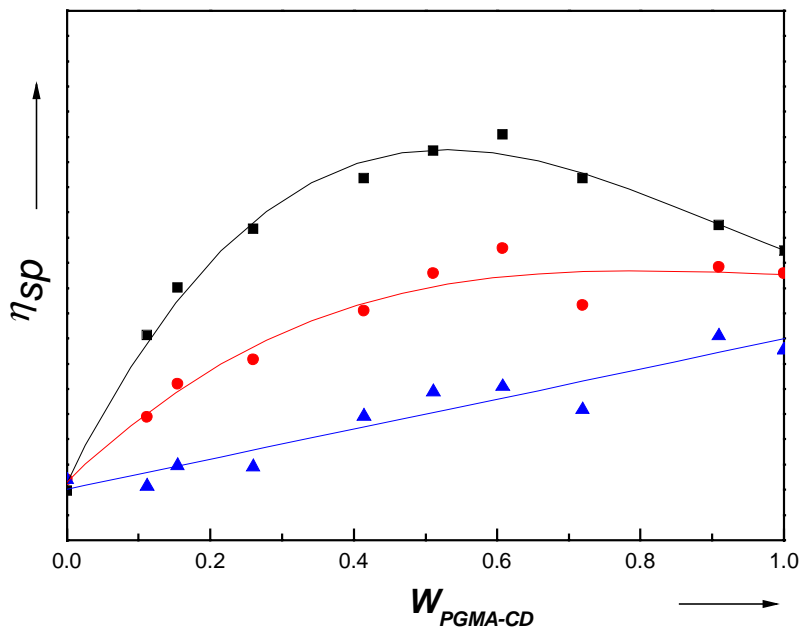

Figure S3. Reduced viscosity of PGMA-CD and PtBA-ADA solution in DMF with a total polymer concentration of $2.0 \mathrm{mg}^{\circ} \mathrm{mL}^{-1}$ as a function of weight fraction of PGMA-CD. The viscosity of mixed solution was measured at $25^{\circ} \mathrm{C}$ (square), $35^{\circ} \mathrm{C}$ (round) and $50{ }^{\circ} \mathrm{C}$ (triangle). 


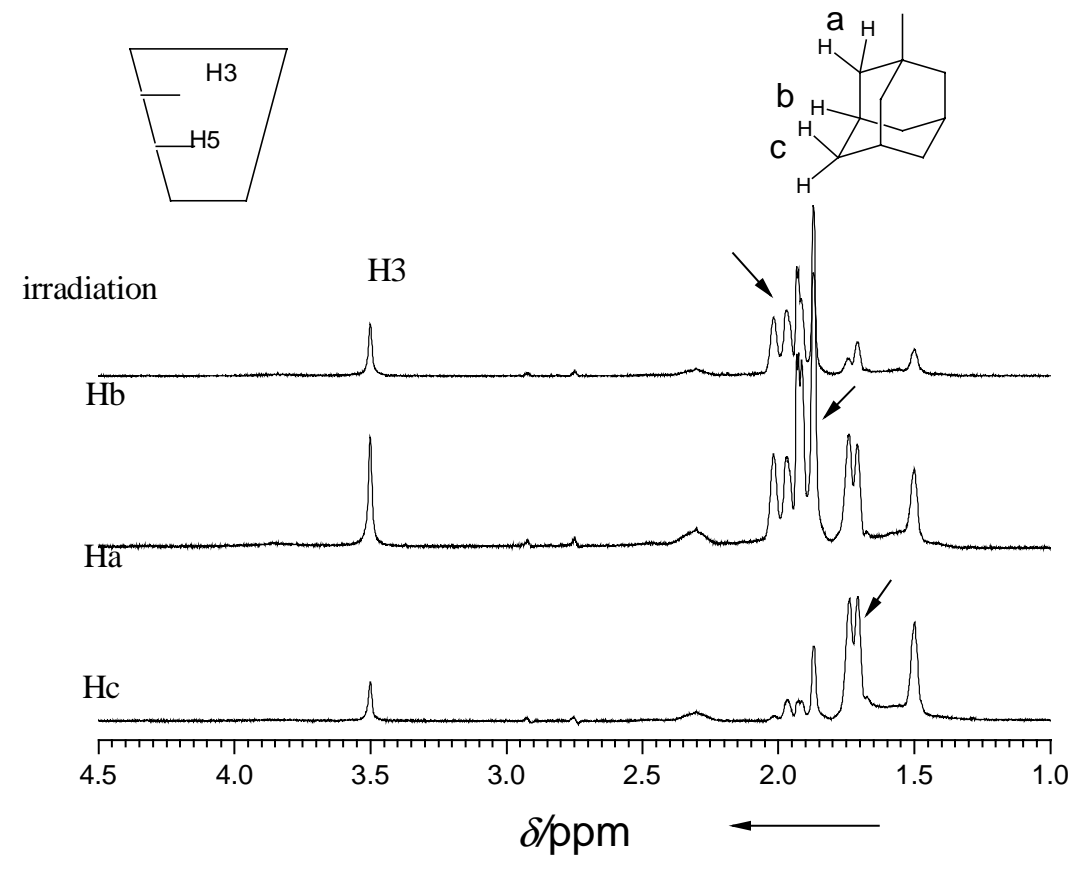

Figure S4. NMR spectra for NOE experiments on PGMA-CD/PtBA-ADA mixture in DMF-d7, Hb (upper), $\mathrm{Ha}$ (middle) and $\mathrm{Hc}$ (lower) of adamantyl groups were irradiated respectively, showing that $\mathrm{H} 5$ of $\beta-\mathrm{CD}$ is too far from the protons of $\mathrm{ADA}(\mathrm{Ha}, \mathrm{Hb}, \mathrm{Hc})$ to present detectable signals.

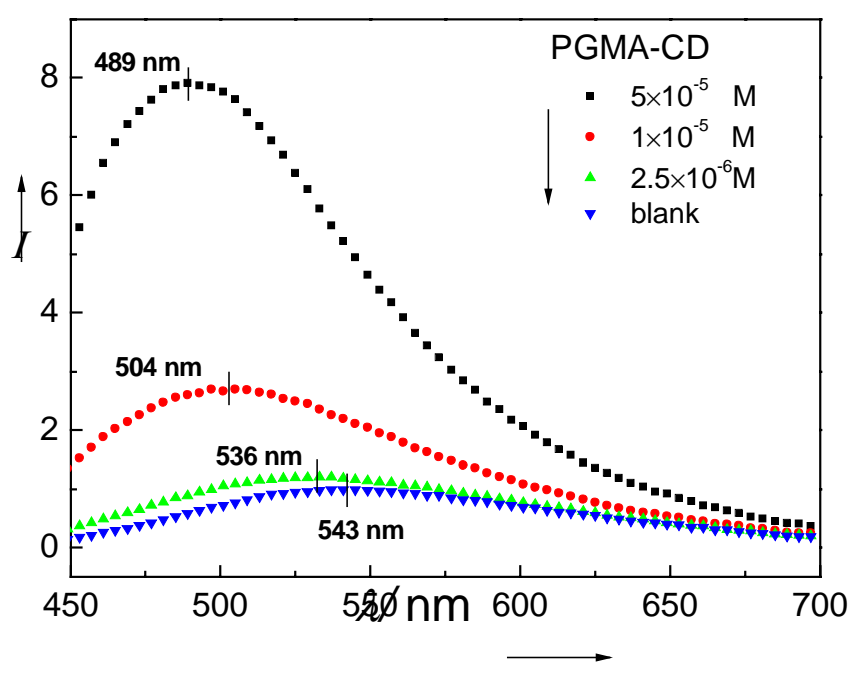

Figure S5 Fluorescence spectra of ANS in water (blue) and in PGMA-CD solutions with different concentrations as indicated. ANS concentration is $5 \times 10^{-5} \mathrm{M}$. Excitation wavelength is $370 \mathrm{~nm}$ and the emission was recorded from 450 to $700 \mathrm{~nm}$. 


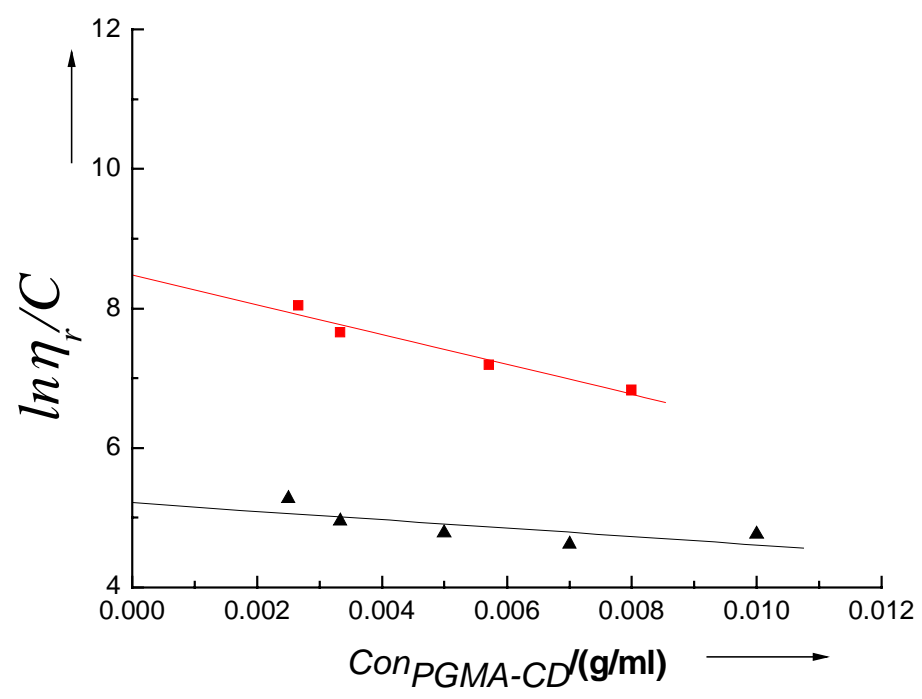

Figure S6. Viscosity measurements of PGMA-CD in water (triangle) and DMF (square) at $30{ }^{\circ} \mathrm{C}$ giving $[\eta]_{\text {water }}=5.21 \mathrm{~mL} \cdot \mathrm{g}^{-1}$ and $[\eta]_{\text {DMF }}=8.48 \mathrm{~mL} \mathrm{~g}^{-1}$

(1) Petter, R. C.; Salek, J. S.; Sikorski, C. T.; Kumaravel, G.; Lin, F. T., J. Am. Chem. Soc. 1990, 112, 3860-3868..

(2) Liu, Y. Y.; Fan, X. D.; Gao, L., Macromol. Biosci. 2003, 3, 715-719. 